PHYSICAL REVIEW B, VOLUME 64, 179903(E)

\title{
Erratum: Magnetic phases of electron-doped manganites [Phys. Rev. B 63, 064431 (2001)]
}

G. Venketeswara Pai

(Published 5 October 2001)

DOI: $10.1103 /$ PhysRevB.64.179903

PACS number(s): 75.30.Vn, 75.30.Gw, 75.30.Et, 71.27.+a, 99.10.+g

In line number 33 of the first column and line number 13 of the second column on page 2 , " $\mathbf{S}_{i}=S_{0}\left(\sin \theta_{i}, \sin \theta_{i}, \cos \theta_{i}\right)$ " should be replaced by " $\mathbf{S}_{i}=S_{0}\left(\sin \theta_{i}, 0, \cos \theta_{i}\right)$." Equation (5) should read

$$
H_{D E}=-J_{H} S_{0} \sum_{j, \alpha} \cos \theta_{j}\left(c_{j \alpha \uparrow}^{\dagger} c_{j \alpha \uparrow}-c_{j \alpha \downarrow}^{\dagger} c_{j \alpha \downarrow}\right)-J_{H} S_{0} \sum_{j, \alpha} \sin \theta_{j}\left(c_{j \alpha \uparrow}^{\dagger} c_{j \alpha \downarrow}+c_{j \alpha \downarrow}^{\dagger} c_{j \alpha \uparrow}\right) .
$$

This was a typographical error and does not affect the results or conclusions. 